\title{
Percutaneous Endoscopic Gastrostomy/ Jejunostomy Combined with Percutaneous Transhepatic Biliary Drainage in Treating Malignant Biliary Obstruction
}

\author{
Jian-Chang Shu ${ }^{a, b}$ Qi-Hong Yang ${ }^{a} \quad$ Xia Lva ${ }^{a}$ Wen-Ru Zhang ${ }^{a}$ Ming-En Li ${ }^{a}$ \\ Xiao-Yan Zhang ${ }^{a}$ Hui-Dong Song ${ }^{a}$ Guo-Rong Ye ${ }^{a}$ Le-Xin Wang ${ }^{b}$ \\ ${ }^{a}$ Department of Gastroenterology, Guangzhou Red Cross Hospital, Jinan University, Guangzhou, PR China; \\ ${ }^{b}$ School of Biomedical Sciences, Charles Sturt University, Wagga Wagga, NSW, Australia
}

\section{Key Words}

Percutaneous endoscopic gastrostomy/jejunostomy ·

Percutaneous transhepatic biliary drainage $\cdot$ Biliary

obstruction · Hepatic function

\begin{abstract}
Objective: To investigate the safety and efficacy of percutaneous endoscopic gastrostomy/jejunostomy (PEG/PEJ) combined with percutaneous transhepatic biliary drainage (PTCD) in treating malignant biliary obstruction. Subjects and Methods: Nine patients ( 6 males and 3 females, average age $71.3 \pm 5.5$ years) with complete obstruction of the biliary tract were treated with PEG/PEJ after PTCD. The PEG/PEJ and PTCD tubes were linked outside of the abdominal wall to direct the externally drained bile back to the jejunum through the PEG/PEJ intestinal tube. Clinical symptoms and liver function were assessed following the treatment. $\boldsymbol{R e}$ sults: The operations were successfully completed in the 9 patients within $40 \mathrm{~min}$ (average $35 \pm 2.9 \mathrm{~min}$ ). Clinical symptoms such as jaundice, abdominal distension, stomachache and diarrhea appeared but improved within 7 days of the operation. Serum levels of bilirubin, aspartate aminotrans-
\end{abstract}

\section{KARGER}

Fax +41613061234 E-Mail karger@karger.ch www.karger.com
(C) 2010 S. Karger AG, Basel

1011-7571/11/0201-0047\$38.00/0

Accessible online at:

www.karger.com/mpp ferase and alanine aminotransferase were reduced $(p<0.01)$ 4 weeks following the treatment. There were no procedural complications. Conclusions: Combined PEG/PEJ and PTCD appeared to be safe and effective in the management of malignant biliary obstruction. Further, larger-scale studies will be needed to verify findings of this report.

Copyright $\odot 2010$ S. Karger AG, Basel

\section{Introduction}

Obstructive jaundice has been associated with decreased quality of life in patients with biliary tract malignancy [1]. Treatment of malignant biliary obstruction depends on the resectability of the underlying neoplasm. In patients who are inoperable due to advanced stages of cancer, drainage catheters or stents have been used in recent years to relieve the symptoms associated with the biliary obstruction [2-5]. The primary purpose of this study was to evaluate the efficacy and safety of percutaneous endoscopic gastrostomy/jejunostomy (PEG/PEJ) combined with percutaneous transhepatic biliary drainage (PTCD) in treating malignant biliary obstruction. 


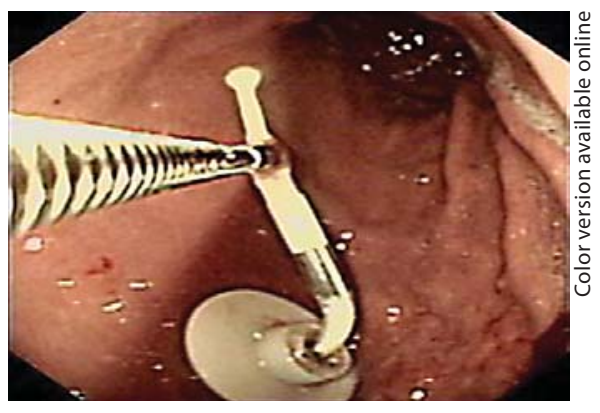

Fig. 1. Under direct observation, the tip of the intestinal tube was grasped while in the stomach as shown and then advanced beyond the ligament of Treitz.

\section{Subjects and Methods}

\section{Patient Recruitment}

This study was approved by the institutional review board of Guangzhou Red Cross Hospital, PR China. Written informed consent was obtained from all participants. Between May 2004 and September 2009, 9 patients ( 6 males, 3 females, average age $71.3 \pm 5.5$ years, range 61-81) who were inoperable for malignant biliary obstruction were recruited to this study. All patients underwent routine physical and laboratory examinations, including blood cell count, prothrombin time, liver and kidney function, electrocardiogram (ECG) and chest X-ray. Computer tomography (CT) examination was carried out to confirm the location of biliary obstruction. Upper gastrointestinal endoscopy examinations were also performed to exclude pyloric obstruction, stomach tumor, or gastric ulcer.

\section{Percutaneous Transhepatic Biliary Drainage (PTCD)}

PTCD was chosen as a primary therapy and performed according to a previously reported method [6]. Under local anesthesia and guided by fluoroscopy, a 20-21G Chiba needle (Nycomed, Paris, France) was inserted into the biliary tract through the space between the right 11th and 12th thoracic vertebrae. Contrast medium was injected through the needle into the biliary tract to display the position and degree of obstruction. When this was achieved, a thin (0.018) guide wire was inserted through the needle into the biliary system, and the needle was removed. A 5F tube was inserted via the guide wire into the biliary tract and the site of obstruction.

The guide wire was then replaced by an ultra-hard guide wire, the $5 \mathrm{~F}$ tube was removed and a 7F biliary drainage catheter (Biosphere Medical, Louviers, France) was passed over the guide wire and placed into the bile ducts. This drainage catheter was flushed with antibiotic once or twice daily. Intravenous antibiotics were also given to all patients for about a week following the insertion of the drainage catheter.

Percutaneous Endoscopic Gastrostomy/Jejunostomy(PEG/PEJ)

Under light sedation, the PEG procedure was performed according to the method reported by Ponsky's group [7, 8]. The PEJ operation was performed on the basis of PEG [9]. After placement of the gastrostomy tube, the PEJ intestinal tube (Freka ${ }^{\circledR}$ PEG Set Gastric FR 15, Fresenius Kabi AG, Bad Homburg, Germany) was inserted into the stomach through the gastrostomy tube. The distal end of the intestinal tube was grasped with a forceps and the endoscope with the forceps in its channel was passed into the duodenal bulb. Under direct observation, the tip of the intestinal tube was grasped while in the stomach as shown in figure 1 and was then advanced to a point below the ligament of Treitz. The $\mathrm{PEJ}$ intestinal tube was fixed to the PEG gastrostomy tube. The PEG/PEJ tube was then connected with the PTCD tube outside of the abdominal wall (fig. 2). Under physiological conditions, bile is excreted into the duodenal lumen through the duodenal papilla. The jejunal extension tube connected with the PTCD tube was beneficial to maintain normal biliary circulation and avoid injuries of the gastric mucosa caused by the bile.

\section{Statistics}

Results were expressed as means \pm SD. Statistical differences were examined by Student's t test. Differences were considered to be statistically significant at $\mathrm{p}<0.05$.

\section{Results}

The complete obstruction of biliary tract was caused by cholangiocarcinoma in 4 patients, pancreas cancer in 4 or liver cancer in 1 . Serum bilirubin was $>179 \mu \mathrm{mol} / 1$ in all patients.

Each operation was completed within 40 min (average $35 \pm 2.9 \mathrm{~min}$ ). Biliary drainage was successfully created in all patients. This was confirmed by the flow of contrast medium from the biliary system to the jejunum through PEJ tubes under X-ray inspection (fig. 3).

There was a significant reduction in serum bilirubin, gamma-glutamyl transferase (GGT), aspartate aminotransferase (AST) and alanine aminotransferase (ALT) within 4 weeks of the procedure (table 1). All patients reported relief from jaundice, abdominal distension, abdominal pain and diarrhea within 7 days of the operation.

No complications were observed during or following the operations. There was no perioperative infection, and no preventative antibiotics were used in these patients. The biliary drainage was examined daily during the hospitalization, and weekly after discharge. The drainage tube was easily assessed by observing the quality and quantity of the discharges, and then contrast media examination was required or attempted. There was no evidence of tube dislodgement or occlusion during the follow-up. The average survival of the patients following the operation was $5.7 \pm 2.1$ months (range 2-10 months). 


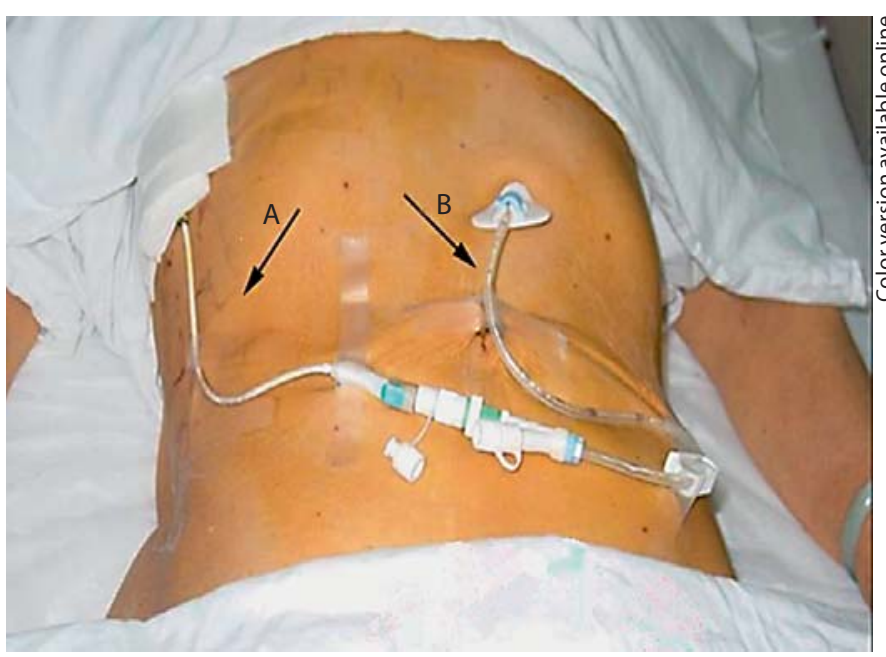

Fig. 2. The PTCD tube (A) was joined with the PEG/PEJ tube (B) outside of the abdominal wall.

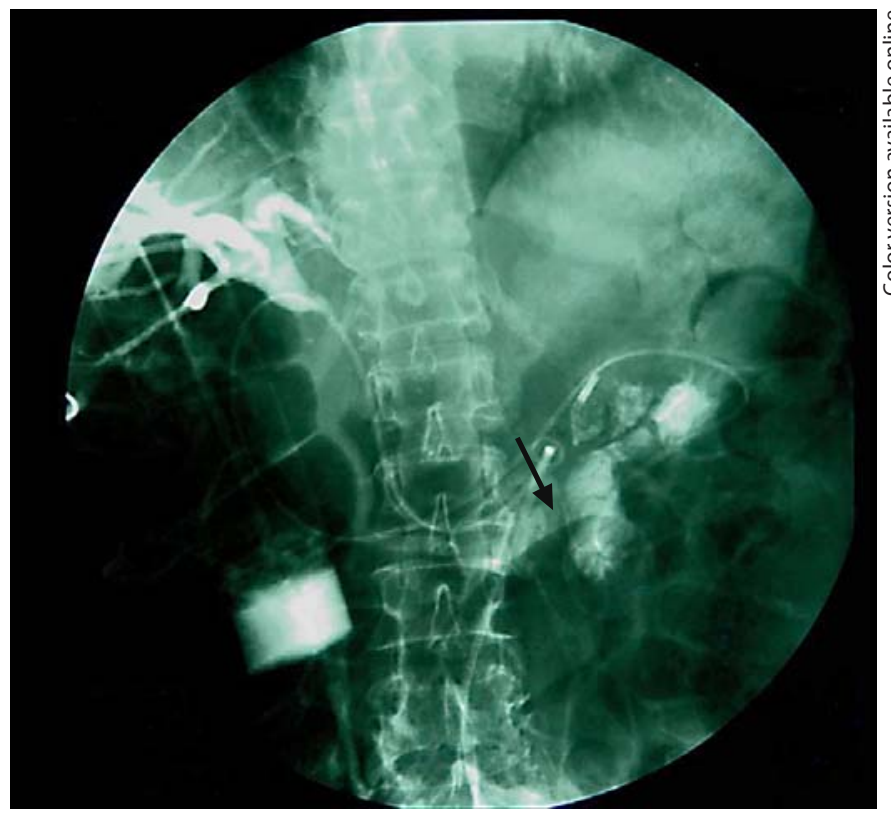

Fig. 3. Under X-ray inspection, successful biliary drainage was confirmed by the flow of contrast medium (arrow) from the biliary system to the jejunum through the PTCD and PEJ tubes.

Table 1. Liver function before and after PTCD and PEJ procedures $(n=9)$

\begin{tabular}{llcccr}
\hline & Before & Week 1 & Week 2 & Week 3 & Week 4 \\
\hline TBil, $\mu \mathrm{mol} / \mathrm{l}$ & $350.8 \pm 34.2$ & $227.9 \pm 28.4$ & $135.5 \pm 22.8$ & $119.3 \pm 19.7$ & $54.9 \pm 16.2^{*}$ \\
GGT, U/l & $369.2 \pm 39.8$ & $291.6 \pm 29.9$ & $173.3 \pm 25.3$ & $127.8 \pm 21.2$ & $124.6 \pm 13.6^{*}$ \\
ALT, U/l & $196.7 \pm 27.7$ & $71.0 \pm 9.5$ & $39.3 \pm 6.2$ & $26.0 \pm 4.6$ & $24.0 \pm 3.4^{*}$ \\
AST, U/l & $166.4 \pm 23.3$ & $54.3 \pm 8.1$ & $45.0 \pm 6.9$ & $44.7 \pm 7.5$ & $44.0 \pm 5.1^{*}$ \\
\hline
\end{tabular}

${ }^{*} \mathrm{p}<0.01$. TBil = Total bilirubin; GGT = gamma-glutamyl transferase; ALT = alanine aminotransferase; AST $=$ aspartate aminotransferase.

\section{Discussion}

The major findings of this study are: in patients with malignant biliary obstruction, PEG/PEJ combined with PTCD provided adequate bile drainage, leading to significant improvement of clinical symptoms; the serum levels of bilirubin were reduced and liver function was improved following this novel treatment; there were no procedural complications such as infection due to drainage tube dislodgment or obstruction during hospitalization or follow-up.

PTCD is a common choice of therapy when surgical or endoscopic therapies were unsuccessful or unsuitable [3-
5]. However, following PTCD, patients have to carry a drainage pack for a long time which is inconvenient. Furthermore, long-term loss of massive bile may cause metabolic disturbances and affect the quality of life of the patients.

Combined PEG/PEJ and PTCD offers several benefits in treating malignant obstruction of the biliary tract: bile circulation route accords with the physiological enterohepatic circulation; the procedure helps with the normal absorption and digestion processes; it reduces the loss of fluid, gastric acid and electrolytes, and it may improve the patient's quality of life through the relief of symptoms associated with biliary obstruction. 
Despite the difficult conditions seen in such patients, thus far no major technical problems have been encountered during tube placement. One of the potential limitations of this study is that the number of patients was relatively small, and the follow-up was relatively short because of the life span constraints on these terminally ill patients. Therefore, the long-term effect of this treatment is unknown. Frequently mentioned contraindications for PEG such as infiltration of the stomach by tumor, ascites or peritoneal carcinosis $[10,11]$ would apply to this combined PEG/PEJ and PTCD therapy. Furthermore, patients with coagulation disturbance will not be suitable candidates for these procedures.

\section{Conclusion}

The combined PEG/PEJ and PTCD procedure appears to be safe and effective in alleviating the symptoms of malignant biliary obstruction. Larger clinical trials are required to confirm the long-term safety and efficacy of this treatment.

\section{References}

1 Pitt HA, Gomes AS, Lois JF, Mann LL, Deutsch LS, Longmire WP Jr: Does preoperative percutaneous biliary drainage reduce operative risk or increase hospital cost? Ann Surg 1985;201:545-553.

-2 Fanelli F, Orgera G, Bezzi M, Rossi P, Allegritti M, Passariello R: Management of malignant biliary obstruction: technical and clinical results using an expanded polytetrafluoroethylene fluorinated ethylene propylene (ePTFE/FEP)-covered metallic stent after 6-year experience. Eur Radiol 2008;18: 911-919.
-3 Yamao K, Bhatia V, Mizuno N, Sawaki A, Ishikawa H, Tajika M, Hoki N, Shimizu Y, Ashida R, Fukami N: EUS-guided choledochoduodenostomy for palliative biliary drainage in patients with malignant biliary obstruction: results of long-term follow-up. Endoscopy 2008;40:340-342.

4 Stern N, Sturgess R: Endoscopic therapy in the management of malignant biliary obstruction. Eur J Surg Oncol 2008;34:313-317.

5 Ishizaki Y, Mitsusada M, Wakayama T: Percutaneous transhepatic biliary drainage combined with percutaneous endoscopic gastrostomy for internal biliary drainage. J Am Coll Surg 1994;179:738-740.

6 Glenn F, Evans JA, Mujahed Z, Thorbjarnarson B: Percutaneous transhepatic cholangiography. Ann Surg 1962;156:451-460.

7 Gauderer MW, Ponsky JL, Izant RJ Jr: Gastrostomy without laparotomy: a percutaneous endoscopic technique. J Pediatr Surg 1980;15:872-875.
8 Ponsky JL, Gauderer MWL: Percutaneous endoscopic gastrostomy: a nonoperative technique for feeding gastrostomy. Gastrointest Endosc 1981;27:9-11.

$\checkmark 9$ Ponsky JL, Aszodi A: Percutaneous endoscopic jejunstomy. Am J Gastrenterol 1984; 79:113-116.

10 Zera RT, Nava HR, Fischer JI: Percutaneous endoscopic gastrostomy (PEG) in cancer patients. Surg Endosc 1993;7:304-307.

11 Adelson MD, Kasowitz MH: Percutaneous endoscopic drainage gastrostomy in the treatment of gastrointestinal obstruction from intraperitoneal malignancy. Obstet Gynecol 1993;81:467-471. 